C2009 IEEE. Personal use of this material is permitted. However, permission to reprint/republish this material for advertising or promotional purposes or for creating new collective works for resale or redistribution to servers or lists, or to reuse any copyrighted component of this work in other works must be obtained from the IEEE. 


\title{
Benchmarking of Virginia Tech GPAs to Curtin University Course Weighted Averages
}

\author{
Euan Lindsay and Richard M. Goff \\ e.lindsay@curtin.edu.au, richgoff@vt.edu
}

\begin{abstract}
Having a single numerical value to represent a student's overall level of achievement is useful. At Curtin University of Technology, it is the Course Weighted Average (CWA) - an average of percentage grades received for each course. At Virginia Tech, it is the Grade Point Average (GPA) - an average of values based upon letter grades for courses (letters initially based upon percentage grades). To facilitate exchanges and/or transfers between U.S., Australian and other institutions, it is useful to benchmark the equivalency of GPAs to CWAs. The difficulty comes through the differences in which grade corresponds to which mark. At Virginia Tech, a mark of $71 \%$ earns a $\mathrm{C}$-, which is a GPA of 1.7; below the 2.0 required for graduation. By contrast, at Curtin a CWA of $71 \%$ results in a degree with honors. This paper reports on a study of cohorts of freshman engineering students at each institution ranked by GPA and CWA, and comparisons drawn at equivalent percentile ranks within each cohort. This yielded an equation to convert GPA to CWA allowing comparable student achievement at the two universities to be evaluated and contributing greatly to facilitating exchanges and/or transfers between U.S. and Australian universities.
\end{abstract}

Index Terms - Comparative grading, Equivalent cohorts, Grade point average, International transfer

\section{INTRODUCTION}

It is useful and convenient in many circumstances to have a single numerical value to represent a student's overall level of achievement. At Curtin University of Technology, this is the Course Weighted Average (CWA) - a numerical average of the percentage grades received for each unit the student attempts. At Virginia Tech, like most US engineering schools, the single value is the GPA - an average of numerical values that are based upon letter grades for units, which are in turn based upon percentage grades within a unit. In order to facilitate exchanges and/or transfers between these institutions and others it would be useful to benchmark the equivalency of GPAs to CWAs.

The difficulty for benchmarking comes through the differences in which grade corresponds to which mark. In the Virginia Tech system, a mark of $71 \%$ earns a C-, which leads to a GPA less than 2.0; where 2.0 is the absolute minimum for graduation [1]. By contrast, a student at Curtin who earns a CWA of $71 \%$ will be given their degree with honors [2].

There are obviously differences in the way in which work is assessed, and these require deep investigations to allow for meaningful benchmarking to occur. The first step is to determine an equivalency for GPA and CWA measurements, so that there can be some clarity as to what constitutes above and below average performance.

There are certainly websites and references that show how to calculate a student's GPA, e.g. [3]. While some Australian universities will explain how to calculate a GPA [4], there is no easily obtainable reference that explains what equivalent marks that GPA would represent in the Australian context.

This paper reports on a study of freshman engineering students in which a cohort of students at each institution were ranked by GPA and CWA, and comparisons drawn at equivalent percentile ranks within the cohort. This comparison allows for a benchmarking conversion of GPA to equivalent CWA and vice versa to be drawn. The results of this research and the development of a quantitative conversion equation to evaluate comparable student achievement will contribute greatly to facilitating future exchanges and/or transfers between U.S. and Australian universities.

The GPA system inherently discretizes the results and limits the range of possibilities at the upper end of achievement. It does not reward the outstanding achiever to the extent that a continuous system such as the CWA does.

Both Virginia Tech and Curtin University are statefunded technology-focused universities with overall enrollments of around 25,000 students, and both have reputations for providing high quality engineering graduates. The authors disagree as to which cohort actually contains the highest achievers. So barring a resolution of this dispute, the assumption of equivalency is made - that is to say that students at equivalent percentile ranks within their cohorts would achieve at the same percentile rank in the alternative institution.

While this assumption is inevitably incorrect to some degree, the extent to which it is inaccurate will be small enough not to jeopardize the comparisons. If the two cohorts are not equivalent, then they are at least comparable.

To begin the comparison, the grading schemes at each university need to be illuminated. At Virginia Tech, outside of a particular course, raw numerical grades are never accumulated or even recorded. These raw numerical grades 
Session W1G

are assigned letter marks which are recorded and converted to grade point values which when accumulated result in a grade point average or GPA based on the conversion scheme shown in Table 1. (Recognizing that individual courses may slightly 'bend' the numerical break points between letter grades.)

TABLE 1: NUMERICAL GRADE TO MARK TO GPA CONVERSION

$\begin{array}{ccc}\text { Grade } & \text { Mark } & \text { GPA } \\ 93 & \text { A } & 4.0 \\ 90 & \text { A- } & 3.7 \\ 87 & \text { B }+ & 3.3 \\ 83 & \text { B } & 3.0 \\ 80 & \text { B- } & 2.7 \\ 77 & \text { C+ } & 2.3 \\ 73 & \text { C } & 2.0 \\ 70 & \text { C- } & 1.7 \\ 67 & \text { D }+ & 1.3 \\ 63 & \text { D } & 1.0 \\ 60 & \text { D- } & 0.7 \\ <60 & \text { F } & 0.0\end{array}$

The "equivalent GPA" formula for converting a numerical grade to grade point average (GPA) is shown in (1) below.

$$
\text { GPA }=\text { numerical grade } / 10-5.3
$$

This formula has its weaknesses. Because there is no possibility of getting a GPA higher than 4.0 regardless of how high the numerical grade is above 93, the formula saturates at $93 \%$. In addition, the "plus" grades map incorrectly. A numerical grade of 77 which results in a $\mathrm{C}+$ gives a GPA of 2.4 using the formula instead of a 2.3 which is the fixed standard.

The formula is a linear empirical relationship. The GPA steps are an attempt to map discrete values onto letter marks and have no particular mathematical basis.

Not all assessment types are equally suited to being reduced down to a single numerical measure. Competency based assessments, for instance, measure students on a wide range of skills simultaneously, and it is potentially dubious to simplify this multi-faceted assessment down to a single number.

Many of the university's mechanisms, however, depend upon using a single metric to categorize student performance. Students with high GPAs (or CWAs) can be acknowledged with Honors degrees and scholarships; students with lower values can be identified for additional support, or possibly counseled regarding their suitability for an Engineering career. With this single metric having such a potential impact, it is important that the metrics used are transferrable between institutions - either directly, or through some kind of calibration. This paper presents an analysis of such a calibration.

\section{DESCRIPTION OF DATASET}

To make the comparison of cohorts, the course weighted averages of a first-year cohort at Curtin University consisting of 252 students and the GPAs of a first-year cohort at Virginia Tech consisting of 1,448 students were gathered. Permission was requested and granted to gather and report these data anonymously by the Human Research Ethics Committee at Curtin University and the Institutional Review Board at Virginia Tech.

Medians and standard deviations were calculated for both sets of data. The calculations show that Virginia Tech GPAs have a median of 3.08, and a standard deviation of 0.74. While Curtin University CWAs have a median of $71.8 \%$, and a standard deviation of $7.19 \%$. If Curtin's CWAs are converted to equivalent GPAs using (1), they have a median of 1.906 , and a standard deviation of 0.719 . These results show a similar standard deviation of scores with a clear offset of roughly one point in GPAs.

To more easily visualize the data, several graphs were generated. Figure 1 illustrates the relationship between GPA and percentile rank within the Virginia Tech data:

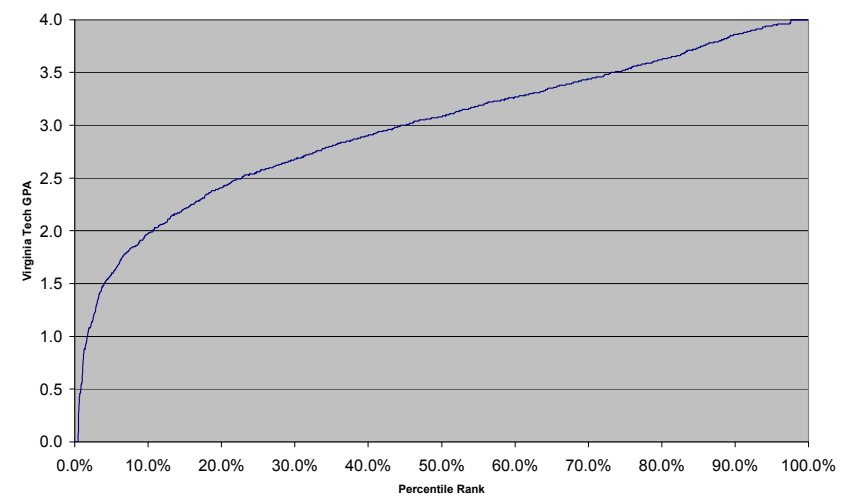

FIGURE 1: VIRGINIA TECH GPA VS PERCENTILE RANK

Figure 2 illustrates the relationship between CWA and percentile rank at Curtin, with Figure 3 illustrating the equivalent GPA vs. percentile rank relationship:

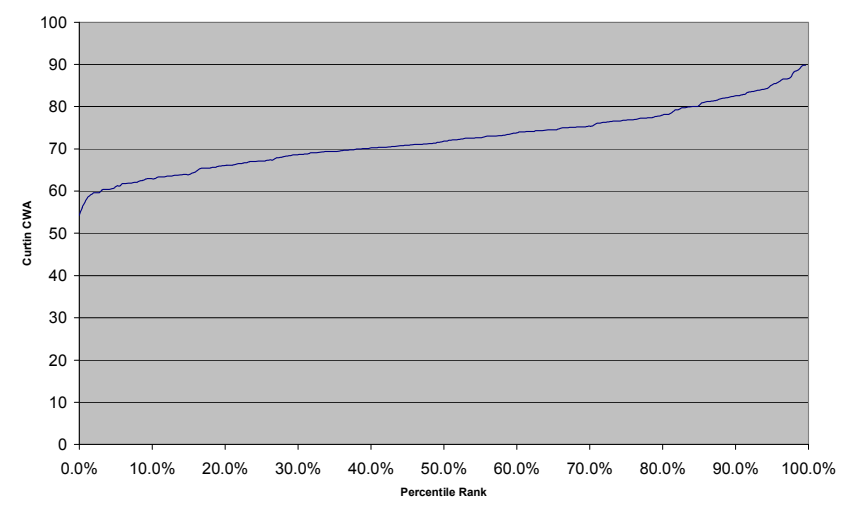

FIGURE 2: CURTIN CWA VS PERCENTILE RANK 
Session W1G

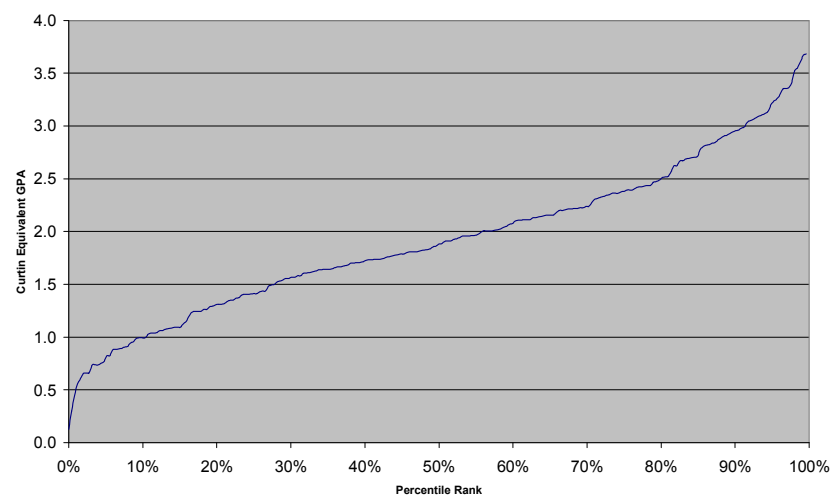

FIGURE 3: CURTIN EQUIVALENT GPA VS PERCENTILE RANK

Figure 3 is a linear rescaling of Figure 2, thus the similarity in shapes; what is noteworthy is the difference in the shapes of the relationships in Figure 1 and Figure 3.

Both cohorts show a sharp drop off at the very low percentiles, and a fairly steady increase throughout the majority of the percentile ranks. There is a substantial difference, however, at the upper percentile ranks. Where the VT data continues its trend, with a saturation of 4.0 GPAs at about the $98^{\text {th }}$ percentile, the Curtin data shows an upswing in CWA above the $80^{\text {th }}$ percentile. A similar upswing is impossible in the VT data -4.0 is the highest mark possible, and once a student earns $93 \%$ they cannot improve their GPA any further.

In addition to the difference in shapes between in Figure 1 and Figure 3, it is also clear that there is a difference in scale. More than half of VT students have a GPA of 3.0 or above; at Curtin only $10 \%$ of the cohort have an equivalent GPA of 3.0. This suggests that there are some substantial differences between the assessments at the two institutions; to explore this thoroughly it is necessary to compare the datasets against each other.

\section{COMPARISON OF DATA}

One of the goals of comparing the two datasets is to determine where a student with a particular GPA (or CWA) stands relative to the rest of their cohort. Table 2 shows the lowest percentile rank at which the key GPA / CWA levels were achieved: Because there is no GPA above 4.0 to account for students who achieve above a $93 \%$ numerical score, two percent of the cohort lives in the 98 percentile range. At Virginia Tech, the $98^{\text {th }}$ percentile is as good as it gets.

\begin{tabular}{|c|c|c|c|}
\hline \multirow[b]{2}{*}{ GPA } & \multicolumn{3}{|c|}{ Curtin } \\
\hline & CWA & Rank & VT Rank \\
\hline 4.0 & $93 \%$ & & 98 \\
\hline 3.7 & $90 \%$ & 99 & 83 \\
\hline 3.3 & $87 \%$ & 96 & 62 \\
\hline 3.0 & $83 \%$ & 90 & 44 \\
\hline 2.7 & $80 \%$ & 83 & 31 \\
\hline 2.3 & $77 \%$ & 73 & 17 \\
\hline 2.0 & $73 \%$ & 53 & 11 \\
\hline 1.7 & $70 \%$ & 36 & 6 \\
\hline 1.3 & $67 \%$ & 22 & 3 \\
\hline 1.0 & $63 \%$ & 9 & 2 \\
\hline 0.7 & $60 \%$ & 2 & 1 \\
\hline
\end{tabular}

Table 2 shows that a VT student with a GPA of 3.3, would be at the $62^{\text {nd }}$ percentile, but a Curtin student with a CWA of $87 \%$ - which are the same raw marks - would be on the $96^{\text {th }}$ percentile within their cohort.

It is clear from Table 2 that the assessment expectations and outcomes are not equivalent at the two institutions. The two cohorts, however, have been assumed to be equivalent, or at least comparable. Building on this assumption, it is therefore meaningful to consider what grades are achieved by students at matching percentiles in the two institutions. Figure 4 (overleaf) shows the VT GPAs and Curtin equivalent GPAs plotted against each other for each decile: 
Session W1G

GPAs vs Percentiles

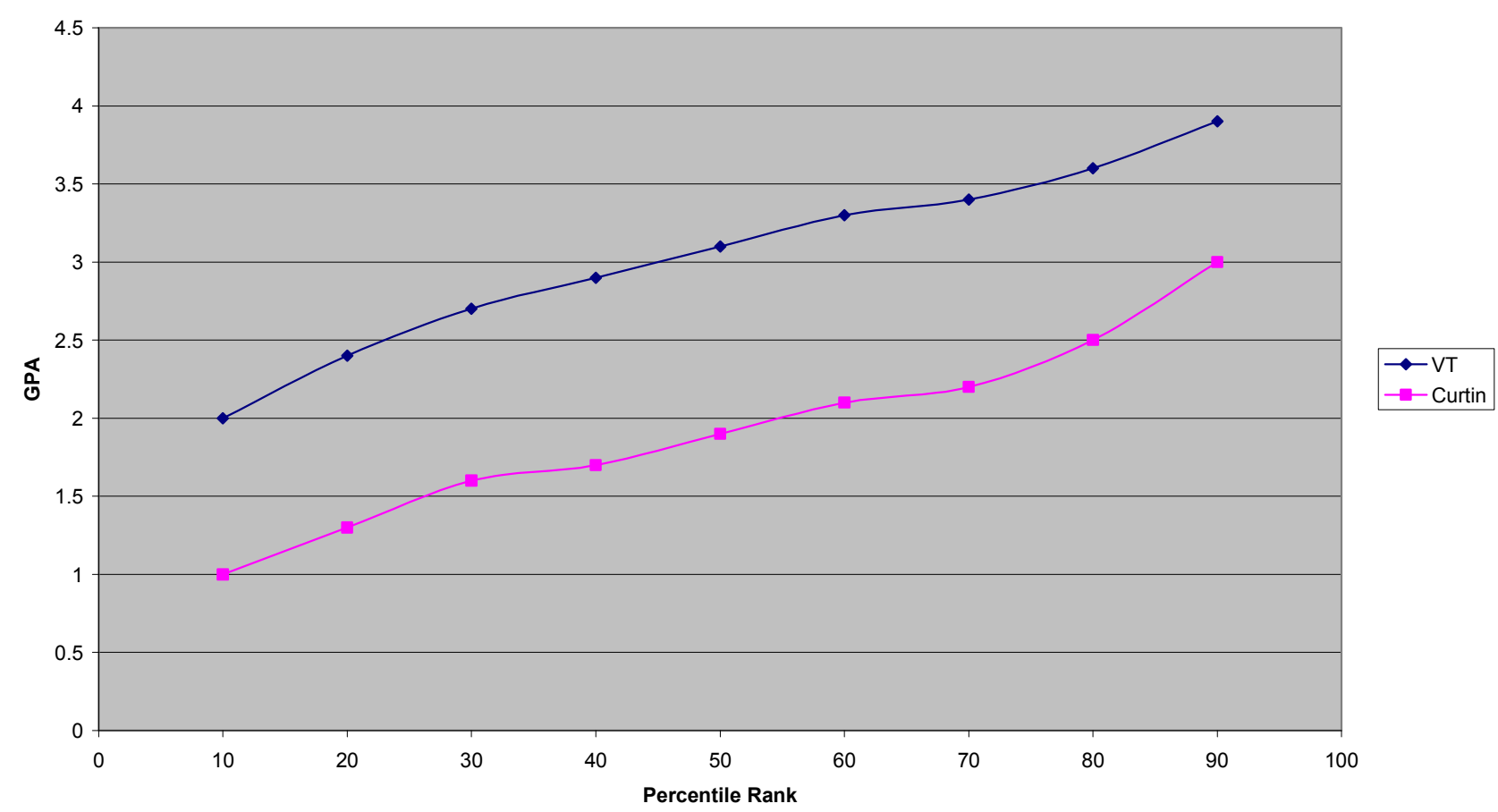

FIGURE 4: GPAS VS PERCENTILE RANK

Figure 4 shows that the trends in the two relationships are similar, with the two lines almost parallel for most of the cohort. This suggests that the overall distribution of students throughout the range of GPAs is similar at the two institutions - a similar range of GPAs is covered, and the distribution of students amongst those GPA levels is similar. What is different is the actual magnitude of that value.

The difference between the two values for a given percentile rank is illustrated in Figure 5:

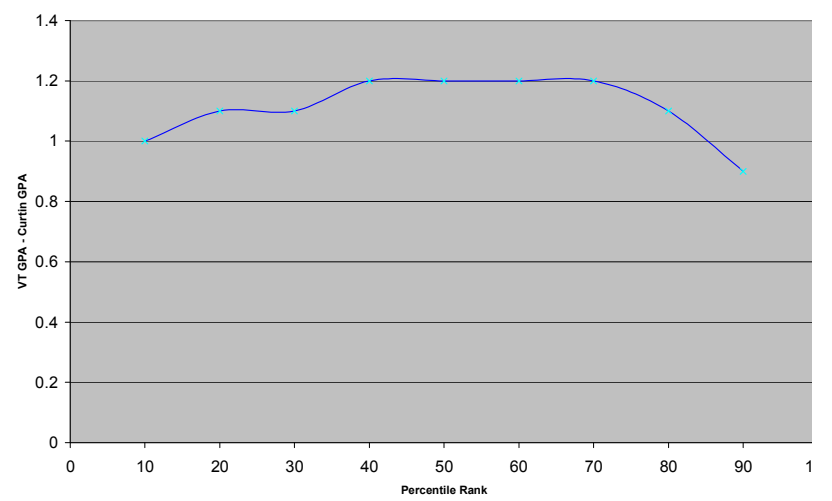

FIGURE 5: DIFFERENCES IN GPAS BY PERCENTILE RANK

Figure 5 shows that the offset between the two GPAs is consistent at a value of about 1.1-1.2. This translates to around an $11-12 \%$ difference in raw marks.

978-1-4244-4714-5/09/\$25.00 @2009 IEEE
Scaling up the Curtin equivalent GPAs by 1.15 and plotting them against the VT GPAs for the deciles produces Figure 6:

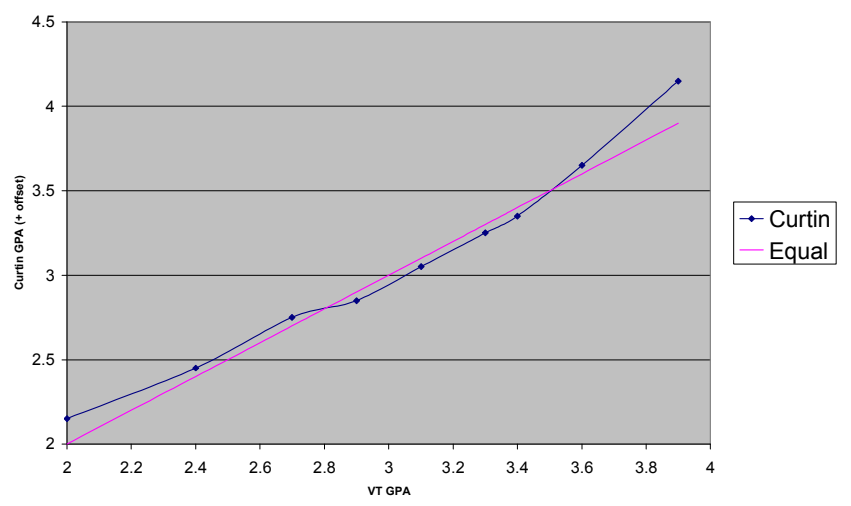

FIGURE 6: CURTIN GPA (W/. OFFSET) VS VT GPA

Figure 6 shows that there is good correspondence between the two GPA measures, with the plot matching closely to the Curtin Offset GPA = VT GPA reference line (Equal). It is worth noting that the Curtin equivalent Offset GPAs exceed 4.0 before the $90^{\text {th }}$ percentile, whereas the VT GPAs do not reach 4.0 until the $98^{\text {th }}$ percentile.

Figure 6 shows that a relatively simple conversion can be used to translate a Curtin equivalent GPA to a VT GPA. Combining this conversion with (1) gives the transformation from Curtin CWA to VT GPA

October 18 - 21, 2009, San Antonio, TX 


$$
\text { VT GPA }=\text { Curtin CWA/10 - 4.15 }
$$

Equation (2) is supported by the empirical data in the midrange and the assumption that the two cohorts are equivalent. It is important, however, to ensure that it is also supported by the nature of the assessment procedures at the two institutions.

\section{DISCUSSION}

It is clear from Figure 4 that there are substantial differences between the assessment outcomes at VT and Curtin; however Figure 6 suggests that there is in fact an underlying correspondence between the ways in which the two institutions assess their students.

It is unlikely that it is just a coincidence that the difference between the two pass marks corresponds closely to the offset required to make the equivalent GPAs match. The two datasets have similar standard deviations, and their medians differ by about the same as the offset suggested by the simplicity of (2).

The two universities have similar assessment practices, with a range of assessments from assignments to exams to ongoing project work. Students are assessed as individuals and in groups; they perform short-term tests as well as extended projects. The key difference is in the range of meaningful grades at each institution. VT operates with a $60 \%$ pass mark, and awards A grades starting at $93 \%$, giving a meaningful range of around $33 \%$. Curtin operates on a $50 \%$ pass mark, and potentially grades students up to $100 \%$, although grades above $90 \%$ are uncommon. As such Curtin has a meaningful range of around $40 \%$.

This offset explains the singularity of the student who averages $71 \%$, which is the median CWA at Curtin. At Curtin, a grade of $71 \%$ is more than halfway through the meaningful range of assessment outcomes. At VT, however, it is only a third.

The two institutions also differ on how they measure the achievement of their highest performing students. VT does not have a metric to distinguish students who average $93 \%$ from those who average $100 \%$ - both will have a GPA that has saturated at 4.0. Curtin, with its pass mark offset, and a continuous range of assessments, can make this distinction. Whether this distinction is useful - other than as a matter of pride for the students - is another question entirely.

Most universities have a scale for academic performance - whether it is a High Distinction / Distinction / Credit / Pass system, a First / Second / Third class Honors system, or an A / B / C / D system, there are different levels of achievement. The positions on these scales are comparable, even if the corresponding numerical values for assessments differ.

For universities operating comparable degree programs, it is simply a matter of unpacking the relationship between raw percentage marks and the corresponding distinction / honor / letter grades, and scaling each student's performance

\section{Session W1G}

relative to the thresholds for each level of performance. In this way differences between pass marks - such as the $10 \%$ difference between Curtin and VT - can be accounted for, and a meaningful equivalent student performance can be determined.

\section{CONCLUSION}

The analysis of the datasets shows that there are substantial differences between the raw assessment outcomes for freshman engineering students at Curtin and at Virginia Tech. A deeper analysis of this data, however, shows that the assessment regimes are actually strongly similar.

When converted into equivalent GPAs, there is a strong relationship between the two datasets, with an offset between the two that corresponds well with the difference in pass marks at the two institutions. The standard deviations of the distribution of grades are also very close, suggesting that the distinctions made between students are also comparable.

The difference appears to be in the mapping of student achievement to a numerical mark. At Curtin, a student must be excellent to achieve a grade above $83 \%$, whereas at VT outstanding achievement is awarded a mark of $93 \%$ or above. The differentiation between students is still present; it is the calibration that differs, as described in (2).

The CWA is not a universal metric - schools can and do use different systems for aggregating student performance. Most Australian schools, however, assess individual units with a percentage mark, and so a CWA equivalent value can be inferred. These values, along with the technique explained in this paper, allow for a meaningful comparison for students at institutions with different assessment schemes to be performed.

\section{REFERENCES}

[1] Virginia Polytechnic Institute and State University. Undergraduate Course Catalog and Academic Policies. [Blacksburg, Va.]: Virginia Tech, 2004.

[2] Curtin University of Technology. Assessment Manual 2008. http://policies.curtin.edu.au/policies/students.cfm

[3] http://www.happyschoolsblog.com/gpa-converter-percentage-to-gpa/

[4] Monash University, http://www.adm.monash.edu.au/servicecentre/gpa-wam.html

\section{AUTHOR INFORMATION}

Euan Lindsay, Department of Mechanical Engineering, Curtin University of Technology, Perth, Australia, e.lindsay@curtin.edu.au

Richard M. Goff, Department of Engineering Education, Virginia Tech, Blacksburg, VA 24061, richgoff@vt.edu 